\title{
Pretreatment Neutrophil-to-Lymphocyte ratio (NLR) and Platelet-to-Lymphocyte Ratio (PLR) as a Predictive Value of Hematological Markers in Cervical Cancer
}

\author{
I Putu Yuda Prabawa ${ }^{1,2 *}$, Agha Bhargah ${ }^{3}$, Firdy Liwang ${ }^{4}$, Deasy Ayuningtyas \\ Tandio ${ }^{5}$, Aditya Leonard Tandio ${ }^{6}$, Anak Agung Wiradewi Lestari ${ }^{2}$, I Nyoman \\ Gede Budiana $^{7}$, Ida Bagus Amertha Putra Manuaba ${ }^{8,9}$
}

\begin{abstract}
Background: Inflammation represents a pivotal role in the progression of cervical cancer. The hematological markers of inflammation in complete blood count (CBC) panel are potentially useful in determining the prognosis of the disease. Objective: The aim of the study was to investigate whether the pretreatment neutrophil-to-lymphocyte ratio (NLR) and platelet-to-lymphocyte ratio (PLR) could be used as in predicting the stage of cervical cancer. Methods: A retrospective cross-sectional study involving 282 patients with cervical cancer was enrolled at Sanglah General Hospital for five years (2013-2017). The histopathological records and complete blood counts (CBC) of the patients were collected and analyzed using SPSS ver. 16 software. FIGO stage I-II and III-IV were classified as early and advance stage respectively. Results: The median NLR and PLR were significantly higher in the advance stage compared with early stage (7.58 (1.36-33.20) and 247.89 (97.10-707.11); p-value $=0.001)$. A strong positive correlation was found between the staging of cervical cancer and NLR $(\mathrm{r}=0.638)$ and PLR $(\mathrm{r}=0.668)$. The AUC, sensitivity, and specificity value of NLR and PLR were $0.803(82 \% ; 71 \%)$ and $0.716(72 \% ; 70 \%)$. Advanced stage of cervical cancer was found in high NLR (adjusted OR: 9.02; 95\%CI=2.42-33.64; $\mathrm{p}=0.001$ ) and PLR (adjusted OR = 2.47; 95\% CI = 1.45-4.85; $\mathrm{p}=0.032$ ). Conclusion: Increased pretreatment NLR and PLR values may provide a useful information in predicting the staging of cervical cancer.
\end{abstract}

Keywords: Cervical cancer- Neutrophil-to-lymphocyte ratio- platelet-to-lymphocyte ratio, staging

Asian Pac J Cancer Prev, 20 (3), 863-868

\section{Introduction}

Cervical cancer (CC) is known as the second most common gynecological malignancy of women worldwide whereas 250,000 patients approximately die from this disease annually (Jemal et al., 2011). The host response to malignant tumors involved in systemic alterations, not only changes in the microenvironment. Several studies has been established that systemic inflammation is involved in the progression of cancer at different stages such as initiation, promotion, invasion, and metastasis (Grivennikov et al., 2010). The systemic inflammation can be manifested as neutrophilia, thrombocytosis and relative lymphocytopenia detected in a complete blood count (CBC) examination (Grivennikov et al., 2010). Hematological biomarkers classified as routine laboratory tests are easily available and affordable. Serum C-reactive protein (CRP), erythrocyte sedimentation rate (ESR), and Glasgow Prognostic Score (GPS) are established as predictive values for numerous malignant tumors (Hefler et al., 2008; McMillan, 2013; Tas and Erturk, 2017). However, these markers were non-specific and may not always be available in secondary health services. Biopsy, as a gold standard, also needs a special facilities rarely established in remote areas. Therefore, $\mathrm{CBC}$ has a potential to be a hematological biomarkers in predicting the cancer progression.

Neutrophils and platelets supply the required bioactive molecules in neoplastic progression by providing angiogenic, epithelial, and stromal growth factors, as well as matrix-remodeling enzymes (Hanahan and Weinberg, 2011; Grivennikov et al., 2010). In addition, the imbalance

\footnotetext{
${ }^{1}$ Master Program in Biomedicine, Faculty of Medicine, ${ }^{2}$ Department of Clinical Pathology, Sanglah General Hospital, ${ }^{3}$ Post Graduate Student, ${ }^{7}$ Department of Obstetrics and Gynecology, Sanglah General Hospital, ${ }^{9}$ Medical and Health Education, Faculty of Medicine, Udayana University, Bali, ${ }^{4}$ Post Graduate Student, Faculty of Medicine, Sam Ratulangi University, Manado, ${ }^{5}$ Public Health Officer, Timor Tengah Utara Health Department, East Nusa Tenggara Province, Indonesia, ${ }^{6}$ Post Graduate Student in Public Health, Faculty of Medicine, Dentistry and Health Sciences, University of Melbourne, Australia, ${ }^{8}$ International Program of Medicine, Taipei Medical University, Taipei, Taiwan.*For Correspondence: yudaprabawa@unud.ac.id
} 
in the innate and adaptive immune systems, as reflects by lymphocytopenia and an impaired $\mathrm{T}$ lymphocytic response compromises the effectiveness of host-tumor immune responses (Canna et al., 2005; Roxburgh et al., 2009). The involvement of the neutrophils, platelets and lymphocytes may represent the neoplastic progression. The progression of the malignancies to the advanced stage are marked with neutrophilia, thrombocytosis and lymphocytopenia. Therefore, the combination of lymphocytes, neutrophils, and platelets has been investigated to be an independent prognostic factor in determining the progression of malignancies (Lee et al., 2013; Smith et al., 2008).

Neutrophil-to-lymphocyte ratio (NLR) and platelet-to-lymphocyte ratio (PLR) are calculated as neutrophil of platelet counts divided by lymphocyte counts. Neutrophils and lymphocytes are known as the primary components of the tumor-related stroma which are associated to local inflammation and immune responses, as well as reflects the balance between pro-tumor and anti-tumor status (Guthrie et al., 2013; Kwon et al., 2012). Moreover, an elevated platelet count indicates the severity of inflammation in cancer by indirectly related to pro-inflammatory mediators (mainly IL-1, IL-2, and IL-6) (Alexandrakis et al., 2003). Several studies recognized that the elevated ratio of peripheral neutrophils-to-lymphocytes (NLR) is related to poor prognostic indicator in various cancers (Guthrie et al., 2013). PLR is also reported to be associated with a poor outcome in different malignant tumors (Hong et al., 2015; Gunaldi et al., 2015). However, there is a paucity of data indicating that elevated pretreatment values of NLR and PLR are associated with unfavorable outcome or poor prognosis in cervical cancer (Zhang et al., 2014). There has not been a consistent conclusion regarding the use of NLR and PLR as predictive markers in cervical cancer (Wang et al., 2013). These discrepancies could be caused by different study designs and small sample sizes. Therefore, the purpose of this study is to investigate NLR and PLR as the hematological markers in predicting the staging of the cervical cancer.

\section{Materials and Methods}

\section{Study Subjects}

The study involved 282 patients from Sanglah General Hospital, Bali, Indonesia, over a five-year period (2013-2017). The patients were admitted with cervical cancer. The data of the cases were collected from medical records. The histopathological records of the patients were examined and classified into adenocarcinoma or squamous-cell carcinoma. The staging of cancer was stated using FIGO stage classification (stage I, II, III, and IV). The patient's completed blood counts prior to any treatment provided were recorded. Patients were excluded if they had received any cervical neoplasia treatment prior to the admission.

Patients were classified in two groups based on the staging: early (stage I-II) and advance (III-IV). Both groups were evaluated based on standard complete blood count (CBC) results using CELL-DYN Ruby System as a multi-parameter automated hematology analyzer.
The demographic data collected include age, number of parity, and aged at menarche. The white blood cells (WBC), haemoglobin, red cell distributing width (RDW), absolute neutrophil count, absolute lymphocyte count, and absolute platelet count were analyzed as hematological parameters of interest. The neutrophil-to-lymphocyte ratio (NLR) was defined as the ratio between absolute neutrophil count to absolute lymphocyte count. The platelet-to-lymphocyte ratio (PLR) was determined as the ratio between absolute platelet counts to absolute lymphocyte count. Basophil-to-lymphocyte ratio (BLR) and monocyte-to-lymphocyte ratio were also investigated in this study.

\section{Statistical Analysis and Ethical Approval}

For statistical analysis, SPSS ver. 16 was used, $\alpha=0.05$. The data distribution was determined by using the Kolmogorov-Smirnov test. When found normally distributed, the variables were described with mean \pm standard deviation. The not normally distributed variables were described with median (IQR). Pearson's correlation test was conducted to determine the partial correlation between the staging of cervical cancer to NLR, PLR, MLR, and BLR. The discriminative role and cut-off values of NLR, PLR, MLR, and BLR was determined using Receiver Operating Curve (ROC) analysis with Area under the Curve (AUC). The cut-off values used to define the sensitivity and specificity of each variables and risk model in multivariate analysis levels. The study was approved by the Ethics Committee of Udayana University Faculty of Medicine and the teaching hospital, Sanglah General Hospital (1335/UN14.2.2/PD/KEP/2018).

\section{Results}

\section{Subject Characteristics}

There was no significant difference found in age between the advance stage group ( $53.71 \pm 9.50$ years-old) and the early stage group $(52.40 \pm 10.16)$. There was no significant difference in the number of parity and the histopathology between the early and the advance stage. Laboratory parameters such as WBC, lymphocytes, neutrophils, platelet, NLR, PLR, BLR, and MLR were significantly higher in the advance stage cancer group compared to the early stage group $(\mathrm{p}<0.05)$. In contrast, hemoglobin was significantly lower in the advance stage group (Table 1).

\section{Correlation of hematology markers with FIGO stage of cervical cancer}

Partial correlation was used to analyze the correlation between blood parameters (NLR, PLR, BLR, and MLR) against FIGO staging of cervical cancer by controlling age, age at menarche, and parity. There was a statistically significant strong positive correlation between NLR $(r=0.638)$ and PLR (0.668) with cervical cancer staging $(\mathrm{p}<0.05)$. These findings illustrate that the increase NLR and PLR will have a tendency to experience higher staging (more advanced disease severity). However, $\operatorname{BLR}(\mathrm{r}=0.362)$ and MLR $(\mathrm{r}=0.399)$ only had a moderate positive correlation to staging from the cervical cancer 
Table 1. Legend?

\begin{tabular}{|c|c|c|c|}
\hline Characteristics & Early $(\mathrm{n}=186)$ & Advance $(\mathrm{n}=96)$ & $\mathrm{p}$ \\
\hline Age (years) & $52.40 \pm 10.16$ & $53.71 \pm 9.50$ & 0.545 \\
\hline Menarche (years) & $12.45 \pm 0.98$ & $12.21 \pm 0.87$ & 0.262 \\
\hline \multicolumn{4}{|l|}{ Parity (n,\%) } \\
\hline 1 & $3(33.3 \%)$ & $6(66.7 \%)$ & 0.72 \\
\hline 2 & $63(63.6 \%)$ & $36(36.4 \%)$ & \\
\hline 3 & $69(71.9 \%)$ & $9(28.1 \%)$ & \\
\hline 4 & $42(66.7 \%)$ & $21(33.3 \%)$ & \\
\hline 5 & $9(60 \%)$ & $6(40 \%)$ & \\
\hline \multicolumn{4}{|l|}{ FIGO Stage $(\mathrm{n}, \%)$} \\
\hline I & $78(41.9 \%)$ & - & \\
\hline II & $108(58.1 \%)$ & - & - \\
\hline III & - & $75(78.1 \%)$ & \\
\hline IV & - & $21(21.9 \%)$ & \\
\hline Histopathology & & & 0.244 \\
\hline Squamous Cell Carcinoma & $111(61.7 \%)$ & $69(38.3 \%)$ & \\
\hline Adenocarcinoma & $75(73.5 \%)$ & $27(26.5 \%)$ & \\
\hline Haemoglobin (g/dl) & $11.48 \pm 1.84$ & $10.56 \pm 1.83$ & $0.030^{*}$ \\
\hline White Blood Cell (109/L) & $8.53 \pm 4.79$ & $12.41 \pm 6.04$ & $0.001 *$ \\
\hline Neutrophil $\left(10^{9} / \mathrm{L}\right)$ & $5.85 \pm 4.71$ & $10.14 \pm 5.88$ & $<0.001 *$ \\
\hline Monosit $\left(10^{9} / \mathrm{L}\right)$ & $0.57 \pm 0.23$ & $0.61 \pm 0.23$ & 0.4 \\
\hline Lymphocyte $\left(10^{9} / \mathrm{L}\right)$ & $1.36 \pm 0.67$ & $1.78 \pm 0.73$ & $0.008^{*}$ \\
\hline Basophill $\left(10^{9} / \mathrm{L}\right)$ & $0.05 \pm 0.03$ & $0.07 \pm 0.05$ & 0.088 \\
\hline Platelet $\left(10^{9} / \mathrm{L}\right)$ & $281.75 \pm 89.1$ & $318 \pm 160.74$ & $0.041^{*}$ \\
\hline NLR & $2.68(0.88-34.62)$ & $7.58(1.36-33.20)$ & $<0.001 *$ \\
\hline BLR & $0.03 \pm 0.01$ & $0.06 \pm 0.06$ & $0.005^{*}$ \\
\hline MLR & $0.36 \pm 0.18$ & $0.53 \pm 0.28$ & $0.001 *$ \\
\hline PLR & $145.40(72.10-533.25)$ & $247.89(97.10-707.11)$ & $0.001^{*}$ \\
\hline
\end{tabular}

*statistically significant $(\mathrm{p}<0.05)$; SD, Standard Deviations; NLR, neutrophil-to-lymphocyte ratio; BLR, basophil-to-lymphocyte ratio; MLR, monocyte-to-lymphocyte ratio; PLR, platelet-to-lymphocyte ratio.

(lower correlation evidence) (see Table 2).

Predictive model of NLR, PLR, BLR, and MLR as hematological markers in the cervical cancer staging

Predictive models were performed through ROC curve analysis on NLR, PLR, MLR, and BLR parameters

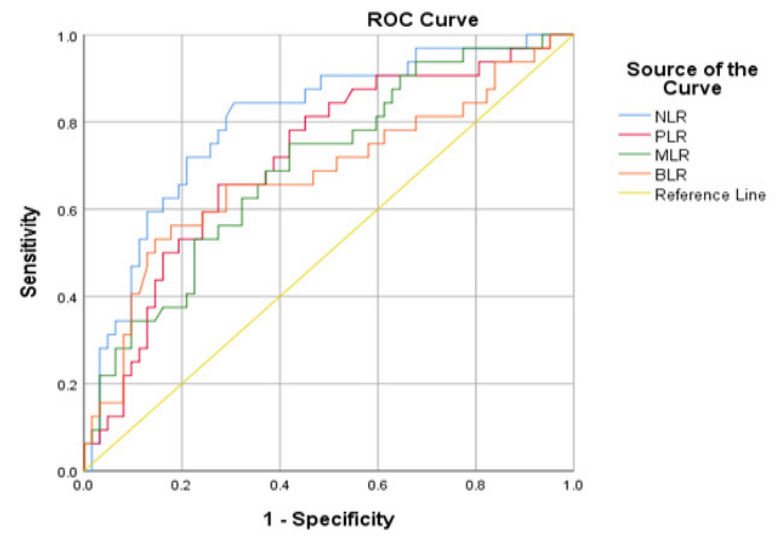

Figure 1. ROC Analysis of NLR, PLR, MLR, and BLR as Predictive Values in the Advance Stage of Cervical Cancer against advance stage of cervical cancer as an outcome in the study (Figure 1). Only NLR and PLR parameters that are feasible to be used as predictive models because they have an area under the curve (AUC $>0.7)$ which is higher than other parameters (BLR and MLR). NLR has a sensitivity value of $82 \%$ and specificity of $71 \%$ with a cut-off value of 3.38. PLR has a sensitivity of $72 \%$ and specificity of $70 \%$ with a cut off value of 172.05 (Table 3 ).

Table 2. Correlation between Hematological Markers with FIGO Stage after Adjustment for Age, Age at Menarche, and Parity

\begin{tabular}{lccc}
\hline Variable & \multicolumn{2}{c}{ FIGO Stage } \\
& $\mathrm{n}$ & $\mathrm{r}$ (correlation coefficient) & $\mathrm{p}$ \\
\hline NLR & 282 & 0.638 & $<0.001^{*}$ \\
PLR & & 0.668 & $<0.001^{*}$ \\
MLR & 0.399 & $<0.001^{*}$ \\
BLR & 0.362 & $<0.001^{*}$ \\
\hline
\end{tabular}

*statistically significant $(\mathrm{p}<0.05) ; \mathrm{NLR}$, neutrophil-to-lymphocyte ratio; BLR, basophil-to-lymphocyte ratio; MLR, monocyte-tolymphocyte ratio; PLR, platelet-to-lymphocyte ratio. 
Table 3. AUC, Cut-off Value, Sensitivity, and Specificity for NLR, PLR, BLR, and MLR in Cervical Cancer Patients

\begin{tabular}{lcccccc}
\hline Parameter & AUC & $95 \%$ CI & Cut-off value & Sensitivity & Specificity & $p$ \\
\hline NLR & 0.803 & $0.70-0.89$ & 3.380 & $82 \%$ & $71 \%$ & $<0.001^{*}$ \\
PLR & 0.716 & $0.60-0.82$ & 172.050 & $72 \%$ & $70 \%$ & $0.001^{*}$ \\
BLR & 0.679 & $0.55-0.80$ & 0.034 & $66 \%$ & $71 \%$ & $0.002^{*}$ \\
MLR & 0.699 & $0.58-0.81$ & 0.409 & $69 \%$ & $63 \%$ & $0.005^{*}$ \\
\hline
\end{tabular}

*statistically significant ( $<<0.05)$; NLR, neutrophil-to-lymphocyte ratio; BLR, basophil-to-lymphocyte ratio; MLR, monocyte-to-lymphocyte ratio; PLR, platelet-to-lymphocyte ratio; AUC, area under the curve; CI, confidence interval.

Table 4. Risk Analysis Model of NLR, PLR, BLR, and MLR in Advanced Cervical Cancer

\begin{tabular}{|c|c|c|c|c|c|c|c|c|}
\hline \multirow[t]{2}{*}{ Univariate Model } & \multirow[b]{2}{*}{ Earlyn (\%) } & \multirow[b]{2}{*}{ Advancen $(\%)$} & \multirow[b]{2}{*}{ OR } & \multirow[b]{2}{*}{$95 \% \mathrm{CI}$} & \multirow[b]{2}{*}{$\mathrm{p}$} & \multicolumn{3}{|c|}{ Multivariate Model } \\
\hline & & & & & & Adjusted OR & $95 \% \mathrm{CI}$ & $\mathrm{p}$ \\
\hline High & $81(58.7 \%)$ & $57(41.3 \%)$ & 12.22 & $6.48-23.01$ & $<0,001^{*}$ & 9.02 & $2.42-33.64$ & $0.001 *$ \\
\hline Normal & $15(10.4 \%)$ & $129(89.6 \%)$ & & & & & & \\
\hline High & $69(48.9 \%)$ & $72(51.1 \%)$ & 4.04 & $2.37-6.90$ & $<0.001^{*}$ & 2.47 & $1.45-4.85$ & $0.032 *$ \\
\hline Normal & $27(19.1 \%)$ & $114(80.9 \%)$ & & & & & & \\
\hline High & $66(47.8 \%)$ & $72(52.2 \%)$ & 3.48 & $2.06-5.87$ & $0.003 *$ & 1.66 & $0.17-7.59$ & 0.56 \\
\hline Normal & $30(20.8 \%)$ & $114(79.2 \%)$ & & & & & & \\
\hline High & $63(53.8 \%)$ & $54(46.2 \%)$ & 4.67 & $2.75-7.91$ & $0,001 *$ & 2.31 & $0.70-7.59$ & 0.165 \\
\hline Normal & $33(20 \%)$ & $132(80 \%)$ & & & & & & \\
\hline
\end{tabular}

*statistically significant ( $\mathrm{p}<0.05)$; NLR, Neutrophil-to-lymphocyte Ratio; BLR, Basophil-to-lymphocyte Ratio; MLR, Monocyte-to-lymphocyte Ratio; PLR, Platelet-to-lymphocyte Ratio; CI, Confidence Interval; OR, Odds Ratio.

Risk analysis model of hematological markers as predictive value in the advance stage of cervical cancer

Risk analysis model is based on the cut-off value of the ROC. NLR, PLR, MLR, and BLR values that exceeded the cut-off point will be classified as high level ratio and values below the cut-off point will be classified in the normal level ratio. In a univariate risk analysis model, all high value of blood parameters (NLR, PLR, MLR, and BLR) are significantly associated as a risk factor for invasive cervical cancer $(\mathrm{OR}>1 ; \mathrm{p}<0.05)$. In the multivariate model, NLR and PLR have a significant association as a risk factor for invasive cervical cancer.

\section{Discussion}

The present study include two morphological types of cervical cancer, squamous cell carcinoma and adenocarcinoma, which might affect the laboratory parameters. The difference in the histology of the cancer could affect the prognosis of the patients. However, this study find no significant difference in the distribution of the histological type between the early and advanced stage group. Moreover, the histological type has been found to have no prognostic information (Mabuchi et al., 2011). Furthermore, the inclusion of two histological type of cervical cancer represents the prevalence of the cervical cancer types in the population where squamous cell carcinoma predominates (Spaans et al., 2018). Hence, this inclusion provide useful information for the use of the laboratory parameters in predicting the prognosis of the commonly found cervical cancer in the secondary health services.

The study found a significant decrease in the hemoglobin levels between early and advance stage patients, Moreover, leukocytosis, neutrophilia, thrombocytosis and lymphocytosis were also observed. However, these difference may be caused by the progression of the cervical cancer disease. Furthermore, these parameters are our focus of investigation to predict the progression of the cervical cancer.

It is established that the inflammatory response contributes to the development of the cancer (Yildirim et al., 2014). The systemic inflammatory response was characterized with the infiltration of leukocytes, such as neutrophil and monocytes, which were attracted by the cytokines and chemokines secreted from the tumor cells (Parida and Mandal, 2014). This response is associated with the rearrangement of the circulating white blood cells like the decreased in the lymphocyte count and the increased in the neutrophil count. This was supported by the Tavares-Murta et al., (2010) study findings. They demonstrated that the incidence of leukocytosis, neutrophilia, lymphopenia, and neutrophil to lymphocyte ratio greater than 5 were significantly higher in the advanced stage of cervical cancer $(\mathrm{p}<0.001, \mathrm{p}<0.05$, $\mathrm{p}<0.001$, and $\mathrm{p}<0.01$ ) (Tavares-Murta BM et al., 2010). A similar finding was found in our study. We observed a higher counts of neutrophil, monocyte and lymphocyte in the invasive than the pre-invasive cancer patients. The Neutrophil Lymphocyte ratio (NLR) found in our study showed a lower cut-off points compare to the previous study. However, the NLR was consistent with the previous finding which is found to be higher in the more severe cancer patients group. Furthermore, the higher Platelet Lymphocyte Ratio (PLR), Basophil Lymphocyte Ratio (BLR) and Monocyte Lymphocyte Ratio (MLR) were found in the invasive cancer patients. Thus, the measurement of the inflammatory response in these 
hematologic parameters would provide the information of the cervical cancer severity.

The use of hematologic parameter in determining the prognosis and managing the therapy for the cervical cancer have become more common among the clinicians (Nakamura et al., 2018). Lee et al., (2012) have suggested the use of NLR for estimating the mortality and the recurrences of cervical cancer. A high NLR is correlated with the poor prognosis of cervical carcinoma patients (Nakamura et al., 2018; Chun et al., 2017; Nakamura et al., 2015). The pretreatment NLR level is inversely correlated with the survival time in the cervical carcinoma patients $(\mathrm{R}=-0.538, \mathrm{R} 2=-0.289, \mathrm{P}=0.002)$. Nakamura et al. found that the progression free and overall survival hazard ratio of 0.317 (95\% confidence interval (CI): 0.167 0.602; $\mathrm{P}<0.001)$ and 0.274 (95\% CI:0.141-0.530; $\mathrm{P}<0.001$ ), respectively (Nakamura et al., 2018). Chun $\mathrm{S}$. et al. found a similar finding with the recurrence free survival hazard ratio of cervical intraepithelial neoplasia for 7.66(95\% CI 2.34-25.1, p=0.001) (Chun et al., 2017). Moreover, the NLR can provide a valid prediction on the prognosis of the patient with stage IIB and the metastasis of the lymph nodes. However, the NLR may have an additional value in diagnosing the severity of the cervical cancer. It is proposed that the NLR can be used as a marker for predicting the cancer invasion (Kose et al., 2015). Although the study has demonstrated a higher NLR in the invasive cancer group than the pre-invasive group, there is no significant correlation between the NLR and the cervical lesion invasion (correlation coefficient $=0.168$, $\mathrm{p}=0.87$ ). This is contrasted with our finding that show a significant correlation between NLR and the FIGO staging of cervical lesion after adjusted for age, age at menarche and the parity. It is possible that the difference in the patient characteristics and the small sample size may affect the correlation between the NLR and the cervical lesion. Furthermore, the NLR has shown a high sensitivity and specificity of $82 \%$ and $71 \%$ with the cutoff of 3.38 in predicting the invasiveness of the cervical cancer. Therefore, further research with a bigger sample size is needed to validate the findings.

The PLR depends on the systemic inflammatory response which is highly affected by the cancer response or other inflammatory condition (Sun et al., 2016). These condition would resulted in the misdiagnosis and ineffective or even harmful therapy. However, the research has demonstrated the association between the PLR and the cervical cancer disease based on the thrombocytosis and lymphocytopenia condition as the inflammatory response (Kose et al., 2015; Smith et al., 2008; Zhou et al., 2014; Chen et al., 2016). It is used as a predictor for the lymph node metastasis in the cervical cancer and improve the risk stratification for predicting the survival. The PLR shows significant reduction in recurrence free and overall survival of cervical cancer $(\mathrm{p}=0.003$ and $\mathrm{p}=0.007$, respectively (Chen et al., 2016). In this study, we found a significant correlation with the FIGO stage after adjusted for age, age at menarche and parity $(\mathrm{p}<0.001)$. A similar finding was observed in another study that reported an inverse correlation between the high pretreatment PLR and the survival of the cervical cancer patients following concurrent chemoradiation therapy $(\mathrm{R}=-0.542, \mathrm{R} 2=-$ 0.294, $\mathrm{P}=0.001$ ) (Nakamura et al., 2015).27 Moreover, the sensitivity and specificity of the PLR in differentiating between pre-invasive and invasive lesion is quite high for $72 \%$ and $63 \%(\mathrm{p}=0.001)$. Furthermore, the PLR provides a simple and readily available test for predicting the severity of cervical cancer (Chen et al., 2016).32 Therefore, the PLR can serve as a simple predictor of cervical cancer invasiveness with cautious interpretation.

The MLR and BLR were considered as a novel parameter for the cervical cancer invasiveness. We believed that this study is the first that explore the association between the MLR, BLR and pre-invasive, invasive cancer disease. There is a significant correlation between these parameters with the FIGO classification of the disease after adjusted for age, age at menarche and parity. However, the sensitivity and the specificity of those were below the NLR and PLR. Thus, the independent use of the MLR and BLR would be less reliable.

However, the univariate analysis for the NLR, PLR, MLR, and BLR for risk model showing a significant association between these parameters and the invasiveness of cervical cancer disease. Although the multivariate analysis showed that the BLR and MLR was not significant after adjustment, it would need further research before it can be ruled out as a potential markers for the cervical cancer invasiveness. This may be resulted from the potential bias due to the small sample size and the retrospective design. Hence, a study with bigger sample size and prospective design can provide more insight on these parameters.

In conclusion, the NLR and PLR showed a potential use for predicting the cervical cancer invasiveness. In addition, the MLR and BLR were observed to be independently associated and correlated with the cervical cancer invasiveness. However, the other inflammatory condition and the limitation of this study may affect the result. Therefore, the use of these parameters in predicting the cervical cancer invasiveness warrant for a more comprehensive and prospective research study before further implementation in the clinical settings.

\section{Conflict of Interest}

The authors declare that they have no financial interests related to the material in the manuscript.

\section{Funding/Support}

The funding was supported by the authors themselves without the involvement of grant, research scholarship, or any other funding sources.

\section{Acknowledgments}

We would like to show our gratitude to Anak Agung Wiradewi Lestari who provided insight and expertise that greatly assisted the research. We are also thankful to Deasy Ayuningtyas Tandio for their comments on an earlier version of the manuscript as well as editorial help. 


\section{References}

Alexandrakis MG, Passam FH, Moschandrea IA, et al (2003). Levels of serum cytokines and acute phase proteins in patients with essential and cancer-related thrombocytosis. Am J Clin Oncol, 26, 135- 40.

Canna K, McArdle PA, McMillan DC, et al (2005). The relationship between tumour T-lymphocyte infiltration, the systemic inflammatory response and survival in patients undergoing curative resection for colorectal cancer. $\mathrm{Br} J$ Cancer, 92, 651-4.

Chen L, Zhang F, Sheng X-G, et al (2016). Peripheral platelet/ lymphocyte ratio predicts lymph node metastasis and acts as a superior prognostic factor for cervical cancer when combined with neutrophil: Lymphocyte. Medicine, 95, e4381.

Chun S, Shin K, Kim KH, et al (2017). The neutrophil-lymphocyte ratio predicts recurrence of cervical intraepithelial neoplasia. J Cancer, 8, 2205-11.

Grivennikov SI, Greten FR, Karin M (2010). Immunity, inflammation, and cancer. Cell, 140, 883-9.

Grivennikov SI, Greten FR, Karin M (2010). Immunity, inflammation, and cancer. Cell, 140, 883-9.

Gunaldi M, Goksu S, Erdem D, et al (2015). Prognostic impact of platelet/lymphocyte and neutrophil/lymphocyte ratios in patients with gastric cancer: a multicenter study. Int J Clin Exp Med, 8, 5937-42.

Guthrie GJ, Charles KA, Roxburgh CS, et al (2013). The systemic inflammation-based neutrophil-lymphocyte ratio: experience in patients with cancer. Crit Rev Oncol Hematol, 88, 218-30.

Hanahan D, Weinberg RA (2011). Hallmarks of cancer: The next generation. Cell, 144, 646-74.

Hefler LA, Concin N, Hofstetter G, et al (2008) Serum C-reactive protein as independent prognostic variable in patients with ovarian cancer. Clin Cancer Res, 14, 710-4.

Hong X, Cui B, Wang M, et al (2015). Systemic immuneinflammation index, based on platelet counts and neutrophillymphocyte ratio, is useful for predicting prognosis in small cell lung cancer. Tohoku J Exp Med, 236, 297-304.

Jemal A, Bray F, Center MM, et al (2011). Global cancer statistics. CA Cancer J Clin, 61, 69-90.

Kose M, Celik F, Kose SK, et al (2015). Could the platelet-to-lymphocyte ratio be a novel marker for predicting invasiveness of cervical pathologies?. Asian Pac J Cancer Prev, 16, 923-6.

Kwon HC, Kim SH, Oh SY, et al (2012). Clinical significance of preoperative neutrophil-lymphocyte versus platelet-lymphocyte ratio in patients with operable colorectal cancer. Biomarkers, 17, 216-22.

Lee S, Oh SY, Kim SH, et al (2013). Prognostic significance of neutrophil lymphocyte ratio and platelet lymphocyte ratio in advanced gastric cancer patients treated with FOLFOX chemotherapy. BMC Cancer, 13, 350.

Lee Y-Y, Choi CH, Kim H-J, et al (2012). Pretreatment neutrophil: lymphocyte ratio as a prognostic factor in cervical carcinoma. Anticancer Res, 32, 1555-61.

Mabuchi S, Matsumoto Y, Isohashi F, et al (2011). Pretreatment leukocytosis is an indicator of poor prognosis in patients with cervical cancer. Gynecol Oncol, 122, 25-32.

McMillan DC (2013). The systemic inflammation-based Glasgow Prognostic Score: a decade of experience in patients with cancer. Cancer Treat Rev, 39, 534-40.

Nakamura K, Nakayama K, Tatsumi N, et al (2018). Prognostic significance of pre-treatment neutrophil-to-lymphocyte and platelet-to-lymphocyte ratios in non-surgically treated uterine cervical carcinoma. Mol Clin Oncol, 9, 138-44.
Nakamura K, Nishida T, Haruma T, et al (2015). Pretreatment platelet-lymphocyte ratio is an independent predictor of cervical cancer recurrence following concurrent chemoradiation therapy. Mol Clin Oncol, 3, 1001-6.

Parida S, Mandal M (2014). Inflammation induced by human papillomavirus in cervical cancer and its implication in prevention. Eur J Cancer Prev, 23, 432-48.

Roxburgh CS, Salmond JM, Horgan PG, et al (2009). Comparison of the prognostic value of inflammation-based pathologic and biochemical criteria in patients undergoing potentially curative resection for colorectal cancer. Ann Surg, 249, 788-93.

Smith RA, Bosonnet L, Ghaneh P, et al (2008). The platelet-lymphocyte ratio improves the predictive value of serum CA19-9 levels in determining patient selection for staging laparoscopy in suspected periampullary cancer. Surgery, 143, 658-66.

Smith RA, Bosonnet L, Ghaneh P, et al (2008). The plateletlymphocyte ratio improves the predictive value of serum CA19-9 levels in determining patient selection for staging laparoscopy in suspected periampullary cancer. Surgery, 143, 658-66.

Spaans VM, Nyoman Bayu Mahendra I, Purwoto G, et al (2018). The landscape of somatic mutations in Indonesian cervical cancer is predominated by the PI3K pathway. Gynecol Oncol, 148, 189-96.

Sun P, Zhang F, Chen C, et al (2016). The ratio of hemoglobin to red cell distribution width as a novel prognostic parameter in esophageal squamous cell carcinoma: a retrospective study from southern China. Oncotarget, 7, 42650.

Tas F, Erturk K (2017). Elevated erythrocyte sedimentation rate is associated with metastatic disease and worse survival in patients with cutaneous malignant melanoma. Mol Clin Oncol, 7, 1142-6.

Tavares-Murta BM, Mendonça MA, Duarte NL, et al (2010). Systemic leukocyte alterations are associated with invasive uterine cervical cancer. Int J Gynecol Cancer, 20, 1154-9.

Wang D, Wu M, Feng FZ, et al (2013). Pretreatment neutrophilto-lymphocyte and platelet-to-lymphocyte ratios do not predict survival in patients with cervical cancer treated with neoadjuvant chemotherapy and radical hysterectomy. Chin Med J (Engl), 126, 1464-8.

Yildirim MA, Seckin KD, Togrul C, et al (2014). Roles of neutrophil/lymphocyte and platelet/lymphocyte ratios in the early diagnosis of malignant ovarian masses. Asian Pac J Cancer Prev, 15, 6881-5.

Zhang Y, Wang L, Liu Y, et al (2014). Preoperative neutrophil-lymphocyte ratio before platelet-lymphocyte ratio predicts clinical outcome in patients with cervical cancer treated with initial radical surgery. Int J Gynecol Cancer, 24, 1319-25.

Zhou X, Du Y, Huang Z, et al (2014). Prognostic value of PLR in various cancers: A meta-analysis. PLoS One, 9, e101119.

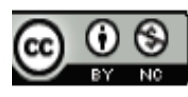

This work is licensed under a Creative Commons AttributionNon Commercial 4.0 International License. 